\title{
Retention of CXCR4 in the endoplasmic reticulum blocks dissemination of a $T$ cell hybridoma
}

\author{
Ingrid S. Zeelenberg, Lisette Ruuls-Van Stalle, and Ed Roos \\ Division of Cell Biology, The Netherlands Cancer Institute, Amsterdam, The Netherlands \\ Address correspondence to: Ed Roos, Division of Cell Biology, The Netherlands Cancer Institute, \\ Plesmanlaan 121, 1066 CX Amsterdam, The Netherlands. \\ Phone: 31-20-5121931; Fax: 31-20-5121944; E-mail: eroos@nki.nl.
}

Received for publication September 14, 2000, and accepted in revised form May 31, 2001.

\begin{abstract}
The dissemination of $\mathrm{T}$ cell hybridomas to multiple nonhematopoietic tissues is blocked by pertussis toxin, suggesting the involvement of a chemokine. To study whether this chemokine is SDF-1, we employed a strategy proposed previously for gene therapy of AIDS, whereby the SDF-1 receptor CXCR4 (also a coreceptor for HIV) is retained in the endoplasmic reticulum (ER) and fails to reach the cell surface. We transfected SDF-1, carrying an ER retention sequence, into a $T$ cell hybridoma. This altered chemokine is retained in the ER, where it binds CXCR4 and prevents the latter protein from reaching the surface. These cells failed to migrate toward SDF-1 or to invade fibroblast monolayers, although they could still migrate toward thymus and activation-regulated chemokine (TARC) and invade TARC-treated monolayers. Furthermore, the ability of the transfected cells to disseminate to multiple organs upon intravenous injection into mice was abolished. This dissemination reflects the in vivo migration patterns of activated and memory $\mathrm{T}$ cells into nonhematopoietic tissues, which is thus likely to depend on CXCR4. Attempts to block CXCR4 function as a therapy for AIDS may affect this migration with consequences for $\mathrm{T}$ cell function. Our results also suggest a decisive role for CXCR4 in the dissemination of hematopoietic malignancies expressing this receptor.
\end{abstract}

J. Clin. Invest. 108:269-277 (2001). DOI:10.1172/JCI200111330.

\section{Introduction}

Activated Tlymphocytes are highly motile and invade hepatocyte and fibroblast monolayers in vitro. Cytotoxic T lymphocytes (CTLs) are similarly invasive, both in the resting and activated state, indicating that after their primary activation, these cells remain constitutively invasive. T cell hybridomas, generated from noninvasive BW5147 T lymphoma cells and activated T cells or CTL clones, are highly invasive $(1,2)$. In contrast to the BW5147 cells, the T cell hybridomas disseminate to many tissues upon intravenous injection, probably reflecting the constitutive migration of activated or memory cells through such tissues (3). We use the $\mathrm{T}$ cell hybridomas as a model to study the mechanisms of this in vivo migration of activated $\mathrm{T}$ cells, which are also likely to be relevant for the dissemination of $\mathrm{T}$ lymphomas and perhaps other hematopoietic malignancies. We have generated mutants and transfectants of these $\mathrm{T}$ cell hybridomas with greatly reduced or completely inhibited dissemination capacity and observed a strict correlation between in vivo dissemination and in vitro invasion in monolayers of embryonic fibroblasts (4-7). Proteins thus shown to be essential for in vivo migration included the adhesion molecule lymphocyte function associated-1 (LFA-1) $\left(\mathrm{CD} 11 \mathrm{a} / \mathrm{CD} 18 ; \alpha_{\mathrm{L}} \beta_{2}\right)$ and $\mathrm{G}_{\mathrm{i}}$ proteins.

The adhesion molecule LFA-1 (CD11a/CD18) plays an essential role in cellular interactions during an immune response and is also involved in the migration of leukocytes into inflamed tissues $(8,9)$. We generated LFA-1-deficient mutants of a $\mathrm{T}$ cell hybridoma, which had lost invasive capacity and did not disseminate, showing that LFA-1 is essential (4). However, LFA- 1 on the T cell hybridomas, as on most leukocytes, is not in an active state; that is, the cells do not adhere spontaneously to the LFA-1 ligand intercellular adhesion molecule (ICAM-1). Thus, LFA-1 needs to be activated before the cells can invade monolayers in vitro or tissues in vivo. Invasion and dissemination were blocked by the transfected $\mathrm{S} 1$ catalytic subunit of pertussis toxin, showing that signals transmitted by $G_{i}$ proteins are required $(5,6)$. This inhibition of invasion was overcome by direct activation of LFA-1 with $\mathrm{Mn}^{2+}$, indicating that one of the effects of this $\mathrm{G}_{i}$ protein signal is the activation of LFA-1 (10).

The requirement for $G_{i}$ proteins implies that a factor acting on a $G_{i}$ protein-coupled receptor is essential for dissemination. The most likely candidates are chemokines, which are small chemoattractant cytokines that regulate trafficking of leukocytes (11). Many chemokines were originally found to control migration of leukocytes during inflammation and are only or mainly expressed in inflamed tissues $(12,13)$. More recently, several chemokines were found that regulate the constitutive migration of leukocytes into and within lymphoid and hematopoietic tissues (14-16). In addition, some chemokines are constitutively expressed in nonhematopoietic tissues. An example is the liver, in 
which stromal cell-derived factor-1 (SDF-1), B cell-attracting chemokine-1 (BCA-1), and liver and activation-regulated chemokine (LARC) are present (17-20). These chemokines are probably involved in the constitutive migration of leukocytes into such tissues. Since the $\mathrm{T}$ cell hybridomas express CXC chemokine receptor 4 (CXCR4), the receptor for SDF-1 (21), we have studied whether this receptor is involved in the in vivo migration of these cells.

In addition to being the receptor for SDF-1, CXCR4 is also a coreceptor for HIV-1 (22). One of the methods proposed to inhibit infection of T cells by HIV-1 is to reduce CXCR4 levels by transfection of an SDF-1 linked to the endoplasmic reticulum (ER) retention signal sequence KDEL, which is retained in the ER (23, 24). Newly synthesized CXCR4 binds to this SDF-1$\mathrm{KDEL}$, is also retained in the ER, and is thus prevented from reaching the cell surface. Clinical trials have been started in which hematopoietic stem cells are transduced with two intrakines, SDF-1-KDEL and RANTES-KDEL (25), the latter to target the second HIV-1 receptor, CC chemokine receptor 5 (CCR5) (26, 27). We have used this same method to reduce CXCR4 surface levels of a $\mathrm{T}$ cell hybridoma. We show here that this causes a complete inhibition of invasion in vitro and of dissemination to multiple tissues in vivo.

\section{Methods}

Cells. The mouse $\mathrm{T}$ cell hybridoma TAM2D2 was generated by fusion of noninvasive BW5147 lymphoma cells with normal activated $T$ lymphocytes (1). The cells were cultured in RPMI-1640 medium with L-glutamine (Life Technologies Ltd., Paisley, United Kingdom) supplemented with $12.5 \mathrm{mM} \mathrm{NaHCO} 3,10 \mathrm{mM}$ 4-(2-hydroxyethyl)-1-piperazineethanesulfonic acid, 10\% NCTC 135 (ICN Pharmaceuticals Inc., Costa Mesa, California, USA), $0.26 \mathrm{~g} / \mathrm{l}$ L-glutamine, $0.05 \mathrm{mM}$ 2-mercaptoethanol, $0.5 \mathrm{mM}$ Na-pyruvate, $1 \mathrm{mM}$ oxaloacetic acid, $0.2 \mathrm{IU} / \mathrm{ml}$ bovine insulin, $100 \mathrm{IU} / \mathrm{ml}$ of penicillin, $100 \mu \mathrm{g} / \mathrm{ml}$ of streptomycin (Life Technologies Ltd.), and 10\% FCS (Life Technologies Ltd.) $(4,28)$. TAM2D2 transfectants were cultured in the same medium supplemented with $1 \mathrm{mg} / \mathrm{ml}$ hygromycin (Calbiochem-Novabiochem Corp., La Jolla, California, USA) or $0.4 \mu \mathrm{g} / \mathrm{ml}$ puromycin (Sigma Chemical Co., St. Louis, Missouri, USA).

Rat embryo fibroblasts (REFs) were cultured in DMEM medium (Life Technologies Ltd.) with 10\% newborn calf serum (Life Technologies Ltd.) and used for invasion assays between passages 5 and 15 .

Generation and transduction of DNA constructs. The SDF1-KDEL construct was generated by PCR with the primers (5'-TAGCTCTAGAGCCATGGACGCCAAG-3' (forward) and $5^{\prime}$-TAGCGAATTCTTACAGCTCGTCCTTCTCGCTCTTGTTTAAAGCTTTCTCCAGGTA- $3^{\prime}$ (reverse with KDEL tag) as described by Chen et al. (23). The construct was cloned into the retroviral vector pLZRSIRES-hyg-EGFP. This vector was based on the pLZRSIRES-zeo vector (29), in which the zeocin resistance
cDNA was replaced by a cDNA encoding a hygromycin resistance-enhanced green fluorescence protein (EGFP) fusion protein (30). The internal ribosomal entry site (IRES) allows the translation of both the KDEL construct and the hygromycin-resistance EGFP fusion protein from one bicistronic mRNA (31). Therefore, high EGFP expression is expected to correlate with high expression of the construct. The vector was transfected by calcium-phosphate precipitation into the virus-packaging cell line $\Phi$ NXE (30). After 8 hours, the medium was refreshed, and 48 hours later the virus supernatant was collected and used to infect the TAM2D2 cells. Three days later, hygromycin was added, and after a few days the selected cells were single-cell FACS-sorted to select clones with high EGFP expression. As a control, the empty vector pLZRS-IREShyg-EGFP was also transfected into TAM2D2 cells.

The SDF(K1R)-KDEL construct was generated as follows. To obtain an arginine instead of a lysine as the first amino acid of the mature protein, the $\mathrm{A}$ at position 65 was mutated to a G. For easier cloning a $\mathrm{C} \rightarrow \mathrm{T}$ mutation was introduced at position 84 to obtain a Cla site. The introduction of the mutations was done by PCR using the forward and reverse primers of the normal SDF-1-KDEL construct and the mutated primers 5 '-CAGCCTGAGCTATCGATGCCCCTGCCG-3' (forward) and 5'-GGGCATCGATAGCTCAGGCTGACTGGTCTACCGTC-3' (reverse). These two PCR products were cloned into the retroviral vector pLZRS-IRES-hyg-EGFP. TAM2D2 cells were transfected and single-cell FACS-sorted to select clones with high EGFP expression.

The TARC-KDEL construct was generated by PCR with the primers 5'-TAGCGAATTCACCATGAGGTCACTTCAGA-3' (forward) and 5'-TAGCTACGTATTACAGCTCGTCCTTCTCGCTCGGCCTTGGGTTTTTCAC-3' (reverse with KDEL tag). The construct was cloned into the retroviral vector pLZRS-IRES-puro-EGFP. This vector was based on the PLZRS-IRES-zeo vector (29), in which the zeocin-resistance cDNA was replaced by a cDNA encoding a puromycin resistance-enhanced EGFP fusion protein (30). TAM2D2 cells were transfected and FACS-sorted to select a bulk population with high EGFP expression.

Binding assay. Cells were washed three times with ice-cold RPMI-1640 and resuspended in RPMI-1640 supplemented with $1 \%$ BSA. Cells $\left(5 \times 10^{5}\right)$ were incubated at $4^{\circ} \mathrm{C}$ in different concentrations of ${ }^{125}$ I-SDF1 (Amersham International, Little Chalfont, United Kingdom) diluted 1:10 with cold SDF-1. To measure aspecific binding an excess of cold SDF-1 was added. After 30 minutes, the cells were washed four times with ice-cold RPMI-1640 supplemented with 1\% BSA. Cells were counted using a gamma counter (Minaxi$\gamma$; Packard, Meriden, Connecticut, USA). Aspecific binding was subtracted from the total binding to calculate the specific binding.

Migration assay. Migration assays were performed as described previously (6). Briefly, transwells with 8- $\mu \mathrm{m}$ 
a

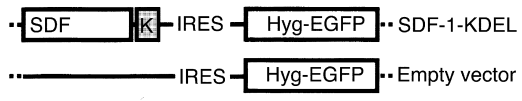

b

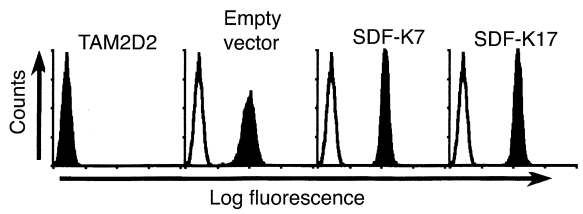

C

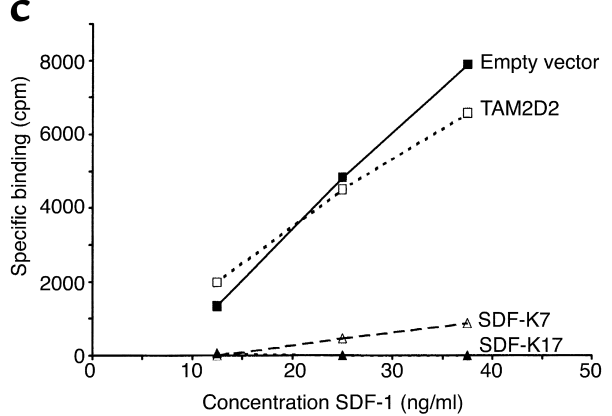

Figure 1

Removal of CXCR4 using an intrakine method. (a) Schematic representation of expression vectors. A cDNA encoding SDF-1 with a Cterminally attached KDEL-sequence was cloned into the retroviral vector pLZRS-IRES-Hyg-EGFP. The empty vector lacks the SDF-KDEL constructs but does contain the IRES sequence and the CDNA encoding the hygromycin resistance-EGFP fusion protein. (b) FACS analysis of EGFP expression of the SDF-1-KDEL clones. Filled histograms are EGFP levels, open histograms are from an untransfected control. Shown are two representative clones out of four. (c) Binding of 125I-SDF to the SDF-1-KDEL clones compared with TAM2D2 cells and the empty vector-transfected control cells. Shown is one representative experiment out of three.

pores were blocked for 2 hours with $0.5 \%$ ovalbumin at room temperature. The lower chamber was filled with $250 \mu \mathrm{l}$ of either SDF-1 or thymus and activation-regulated chemokine (TARC) (PeproTech Inc., Rocky Hill, New Jersey, USA) at $100 \mathrm{ng} / \mathrm{ml}$ in RPMI-1640 supplemented with $0.1 \%$ ovalbumin. The transwells were placed on top, and $10^{5}$ cells washed with ice-cold serum-free medium were added to the upper chamber. After incubation for 2 hours at $37^{\circ} \mathrm{C}$ and $5 \% \mathrm{CO}_{2}$, the migrated cells in the lower chamber were counted.

Invasion assay. Invasion assays were performed as described previously (28). Briefly, confluent REF monolayers in 24-well plates and TAM2D2 transfectants were washed, and the latter were added to the monolayers in serum-free medium. After incubation for 1 hour at $37^{\circ} \mathrm{C}$ and $5 \% \mathrm{CO}_{2}$, the monolayers were extensively washed and fixed with $2 \%$ paraformaldehyde. The invaded cells were counted using phase-contrast microscopy. In some experiments the REF monolayers were pretreated with $100 \mathrm{ng} / \mathrm{ml}$ TARC for 1 hour at $37^{\circ} \mathrm{C}$, and then extensively washed before addition of the TAM2D2 cells.
Dissemination. TAM2D2 cells or transfectants $\left(5 \times 10^{5}\right)$ in $0.2 \mathrm{ml}$ PBS supplemented with $1 \mathrm{mM} \mathrm{CaCl}_{2}$ and 1 $\mathrm{mM} \mathrm{MgCl} 2$ were injected into a tail vein of syngeneic 2to 3-month-old AKR mice or $\mathrm{BALB} / \mathrm{c}$ nude mice. Autopsies were performed when animals were moribund or after 100 days and examined macroscopically and microscopically for the presence of metastases. As a control, $10^{6}$ cells were injected subcutaneously to determine the ability of the cells to grow in vivo. As soon as a minor tumor was visible, the size of the tumor was measured, then measured subsequently every 2 days to establish the proliferation rate.

$R T-P C R$. The organs of a BALB/c nude mouse were frozen in liquid nitrogen and total RNA was extracted using Ultraspec RNA (Tel-Test Inc., Friendswood, Texas, USA). RNA $(5 \mu \mathrm{g})$ was reverse transcribed with oligo-dT primers using the Superscript preamplification system (Life Technologies Ltd.). RT-PCR was performed on the cDNAs from the different mouse tissues with SDF-1-specific primers. Amplification of $\beta$-actin RNA was performed simultaneously using actin-specific primers. Amplified products $(15 \mu \mathrm{l})$ were electrophoresed on a $1.5 \%$ agarose gel.

\section{Results}

Reduction of cell surface CXCR4. To study the role of CXCR4 in T cell hybridoma invasion and dissemination, we used a "intrakine" approach described previously $(23,24)$ to reduce cell surface levels of the chemokine receptor. This approach comprises the transfection of the CXCR4 ligand SDF-1 with a C-terminally attached KDEL sequence. This KDEL sequence binds to the KDEL receptor in the ER and thus retains proteins in the ER. The "intrakine" SDF-1-KDEL binds to newly synthesized CXCR4 and thus prevents it from transport to the cell surface, leading to reduced CXCR4 cell surface levels. To select cells with sufficiently high SDF-1-KDEL expression, we used a retroviral vector in which an IRES (31) was present downstream of the SDF-1-KDEL cDNA, followed by a cDNA encoding a fusion protein of the hygromycin-resistance protein and EGFP. The constructs used are shown in Figure 1a. Both SDF-1-KDEL and the fusion protein are expressed from one bicistronic mRNA, and the expression levels are likely to correlate.

We selected high expressor clones by single-cell FACS sorting, based on their EGFP expression (Figure 1b). The results obtained with two selected clones (SDF-K7 and SDF-K17) are shown, but other clones behaved similarly. To examine whether the SDF-1KDEL construct did reduce CXCR4 surface levels, a binding assay was performed with ${ }^{125}$ I-labeled SDF-1. As shown in Figure 1c, the clones SDF-K7 and SDFK17 bound virtually no SDF-1.

Migration of the SDF-1-KDEL transfectants. The loss of cell surface SDF-1 receptor of the clones SDF-K7 and SDF-K17 was confirmed using a chemotaxis assay. TAM2D2 cells do not migrate at all when no chemokine is present in the lower compartment, but 

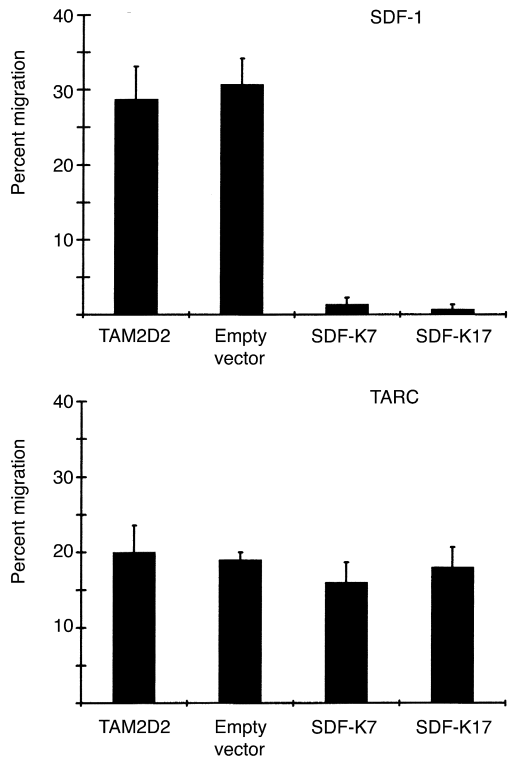

\section{Figure 2}

Migration toward SDF-1 and TARC of SDF-1-KDEL transfectants. Data are percentages of cells that have migrated in 2 hours through a filter to the lower chamber of a transwell containing $100 \mathrm{ng} / \mathrm{ml}$ of the chemokine. Data are averages \pm SEM of three experiments performed.

respond very strongly to the chemokine SDF-1. Whereas SDF-1 is a potent attractant for the untransfected TAM2D2 cells and the empty vector transfectants, the two clones SDF-K7 and SDF-K17 did not migrate at all (Figure 2). Similar results were obtained with the other clones (data not shown). To show the specificity of this effect, we performed a chemotaxis assay with another chemokine: thymus and activationregulated chemokine (TARC) (32). The migration toward TARC was the same as for the untransfected TAM2D2 cells and the empty vector transfectants (Figure 2). This shows that the migratory capacity of the cells is not generally affected, but that the response to SDF-1 is specifically impaired.

Invasion of the SDF-1-KDEL transfectants. In previous studies, a strict correlation was observed between in vivo dissemination of the $\mathrm{T}$ cell hybridomas and in vitro invasion into monolayers of embryonic fibroblasts (4-7). Therefore, this invasion assay appears to be a suitable model to study signal transduction pathways involved in dissemination. Both invasion and dissemination are dependent on $G_{i}$ proteins, since both were completely inhibited by pertussis toxin $(5,6)$. This implies that a $G$ protein-coupled receptor is involved, most likely a chemokine receptor. The embryonic fibroblasts used for the invasion assay express the chemokine SDF-1 in a manner similar to cells in many tissues in vivo (17). Therefore, it seemed conceivable that CXCR4 plays a role in the invasion of the $\mathrm{T}$ cell hybridoma. Indeed, the invasion of the two clones was completely blocked (Figure 3). To again exclude the possibility of a more general effect, we examined whether triggering of another chemokine receptor could restore the invasive capacity. The REF monolayer was pretreated for 2 hours with TARC. This increased the invasion of the control cells from $30-40 \%$ to $35-45 \%$ of added cells. Similarly, the invasion of the clones SDF-K7 and SDF-K17 increased from 0 to $6 \%$ of added cells. This demonstrates that the invasive capacity of the clones is not blocked, although TARC is apparently less efficient in inducing invasion than SDF-1. The effect of the SDF-1-KDEL transfection is therefore specific for CXCR4, showing that invasion completely depends on its ligand SDF-1.

Dissemination of the SDF-1-KDEL transfectants. Having established that SDF-1-induced migration and invasion of the SDF-K7 and SDF-K17 clones were completely blocked, we next tested the in vivo dissemination. We injected $5 \times 10^{5}$ cells into the tail veins of nude mice. The survival curve is shown in Figure 4. The five mice injected with the empty vector transfectants died within 4 weeks with the same organ distribution pattern as observed for TAM2D2 cells in previous studies, that is, massive invasion of liver, spleen, kidneys, and ovaries $(1,2,6)$. In contrast, 18 of the 19 mice injected with either of the two SDF-1-KDEL transfectants survived for 100 days, and upon macroscopic and microscopic examination, no abnormalities were found. Only one mouse, injected with the SDFK7 transfectant, was moribund after 8 weeks, but no tumor was visible in the spleen and kidney. However, a large tumor was found in the retroperitoneal fat and small tumors in the hilus area of the liver. Cells isolated from the large tumor were tested for EGFP expression. EGFP expression was almost completely lost, and therefore it is likely that the expression of SDF-1KDEL was also lost, so this is probably the reason that dissemination did occur. To establish that all cell lines were able to grow in vivo, $10^{6}$ cells were injected subcutaneously, and the size of the tumor was followed over time. The size of all tumors increased 2.4 times a day, showing that the reduced metastatic capacity of the SDF-1-KDEL transfectants cannot be attributed to rejection or impaired in vivo growth.

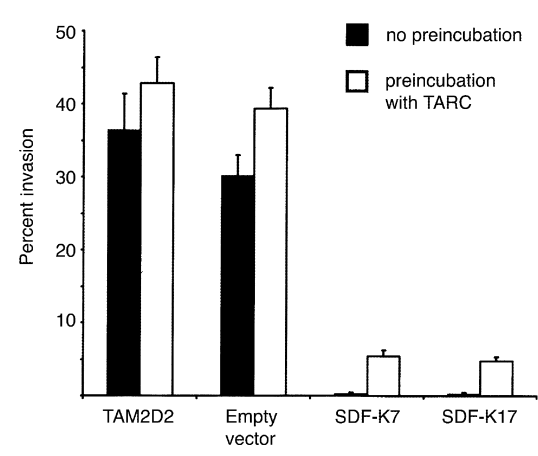

Figure 3

Invasion of SDF-1-KDEL clones into REF monolayers, either pretreated or not pretreated with $100 \mathrm{ng} / \mathrm{ml}$ TARC. Data are the percentages of cells that have invaded within 1 hour and are averages \pm SEM of five experiments performed. 


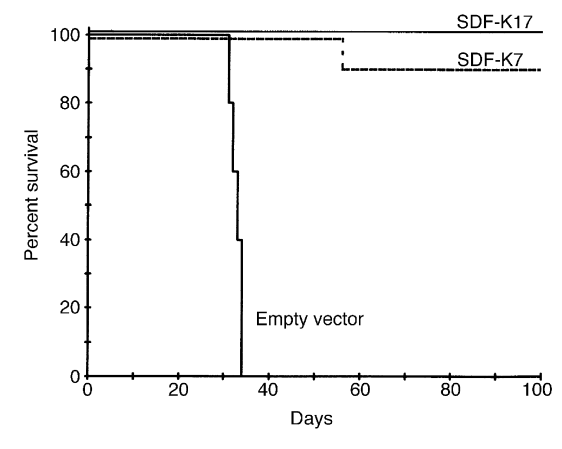

Figure 4

Dissemination of SDF-1-KDEL clones. Shown are survival curves of nude mice injected intravenously with $5 \times 10^{5}$ cells. Mice were killed when moribund or after 100 days. Five mice were injected with empty vector transfectants and ten and nine mice were injected with the SDF-K7 and SDF-K17 transfectants, respectively.

Nonsignaling mutant. Retention of a chemokine in the ER might have undesired effects. Although unlikely, it is imaginable that binding of the SDF-1-KDEL protein to CXCR4 in the ER induces signals that might, for example, lead to downregulation of essential pathways. To exclude this possibility, we introduced a mutation in the SDF-1-KDEL construct to change the first amino acid of the mature peptide from a lysine to an arginine (Figure 5a). This mutation changes the chemokine from an agonist into a nonsignaling antagonist (33). Clones with high $\mathrm{SDF}(\mathrm{K} 1 \mathrm{R})-\mathrm{KDEL}$ expression were selected in a manner similar to those expressing the nonmutated SDF-1-KDEL. The T cell hybridoma was transfected with this mutant SDF(K1R)-KDEL, and transfectants were single-cell FACS-sorted to select clones with high EGFP expression (Figure $5 \mathrm{~b}$ and data not shown). Four clones were obtained with comparable expression. This was, however, only $50 \%$ of the level of the nonmutated SDF-1-KDEL transfectants described above (Figure 1b). As a result, CXCR4 may not be completely retained in the ER, so that low levels of receptor may still be present on the surface of the cells. Indeed, as shown in Figure $5 c$, the clones $\mathrm{SDF}(\mathrm{K} 1 \mathrm{R})-\mathrm{K} 10$ and $\mathrm{K} 30$ were still able to bind ${ }^{125}$ I-SDF-1, approximately one third of the amount bound by TAM2D 2 cells and the empty vector transfectants. As shown in Figure 6a, the chemotaxis of two of the SDF(K1R)-KDEL clones toward SDF-1 was also reduced but not totally blocked. Results obtained with the other clones were similar (data not shown). In the invasion assay, the reduction was quite substantial, but, again, invasion was not completely blocked (Figure 6b and data not shown).

The two clones SDF(K1R)-K10 and SDF(K1R)-K30 were injected into the tail veins of eight and five syngeneic AKR mice, respectively. The survival curve is shown in Figure 6c. All five mice injected with the $\mathrm{SDF}(\mathrm{K} 1 \mathrm{R})-\mathrm{K} 30$ transfectant survived. After 100 days, the mice were examined macroscopically and microscopically and no abnormalities were found. Seven out of the eight mice injected with clone SDF(K1R)-K10 died within 100 days, but much later than mice injected with con- trol cells. Two of these mice had metastases in the hind leg, which had been seen occasionally in previous experiments with normal TAM2D2 cells. The other five mice were all female and had large ovary tumors, besides a small tumor in the liver. The EGFP expression in the cells derived from the tumors was in all cases lower than in the cells at the time of injection. This suggests that the expression of $\mathrm{SDF}(\mathrm{K} 1 \mathrm{R})-\mathrm{KDEL}$ was less stable in clone 10 compared with clone 30 , so that enough CXCR4 reappeared on the surface to enable the cells to disseminate.

TARC-KDEL. Conceivably, the expression of a chemokine with an ER retention signal sequence (KDEL) could have nonspecific effects, due only to the KDEL-sequence and not to the retention of the CXCR4 chemokine receptor in the ER. To exclude this possibility, another intrakine was transduced into the TAM2D2 cells: TARC-KDEL. This intrakine should retain CCR4, the receptor of TARC, inside the cell and therefore block migration toward TARC, whereas migration toward SDF-1 should still be normal. The expression levels of EGFP were comparable to those of the SDF-K7 and SDF-K17 clones, therefore also the expression of the KDEL constructs are likely to be similar. As seen in Figure 7, the TARC-KDEL transfectants have completely lost the capacity to migrate toward TARC, but they do migrate towards SDF-1, showing that the effect of the TARC-KDEL intrakine is specific.

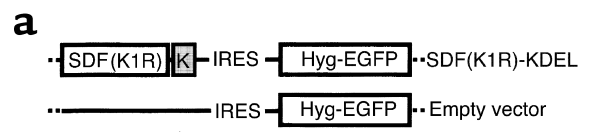

b
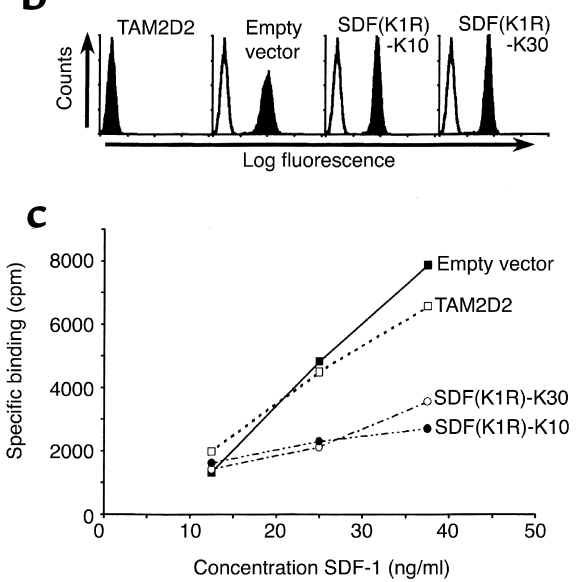

Figure 5

Reduction of CXCR4 surface levels by the nonsignaling mutant SDF1-KDEL, SDF(K1R)-K. (a) Schematic representation of expression vectors. The first amino acid of the mature SDF-1 protein (K) was mutated to an arginine (R) to obtain a nonsignaling SDF-1 and linked to a KDEL sequence. The CDNA was expressed using the retroviral vector pLZRS-IRES-Hyg-EGFP. (b) FACS analysis of EGFP expression of $\mathrm{SDF}(\mathrm{K} 1 \mathrm{R})-\mathrm{KDEL}$ clones. Filled histograms are EGFP levels, open histograms are from an untransfected control. Shown are two representative clones out of five. (c) Binding of 125 I-SDF to mutated SDF(K1R)KDEL clones as compared with TAM2D2 cells and the empty vector tranfectants. Shown is one representative experiment out of three. 
In the invasion assay the TARC-KDEL transfectants behaved in a manner similar to the control cells, with only a slight decrease in invasive capacity $(80 \%$ of the control cells). To investigate the metastatic capacity of the TARC-KDEL transfectant, six mice were injected intravenously. Four mice died within 6 weeks, as seen for the cells transduced with the empty vector. The four mice showed the same metastatic pattern as seen for the control mice with large tumors in liver and spleen. The other two mice were killed after 6 weeks, and no visible metastases were found.

Sites of dissemination. The $\mathrm{T}$ cell hybridomas spread mainly to liver, spleen, kidney, and ovaries, but metastases in other tissues have also been found (2). The sur-
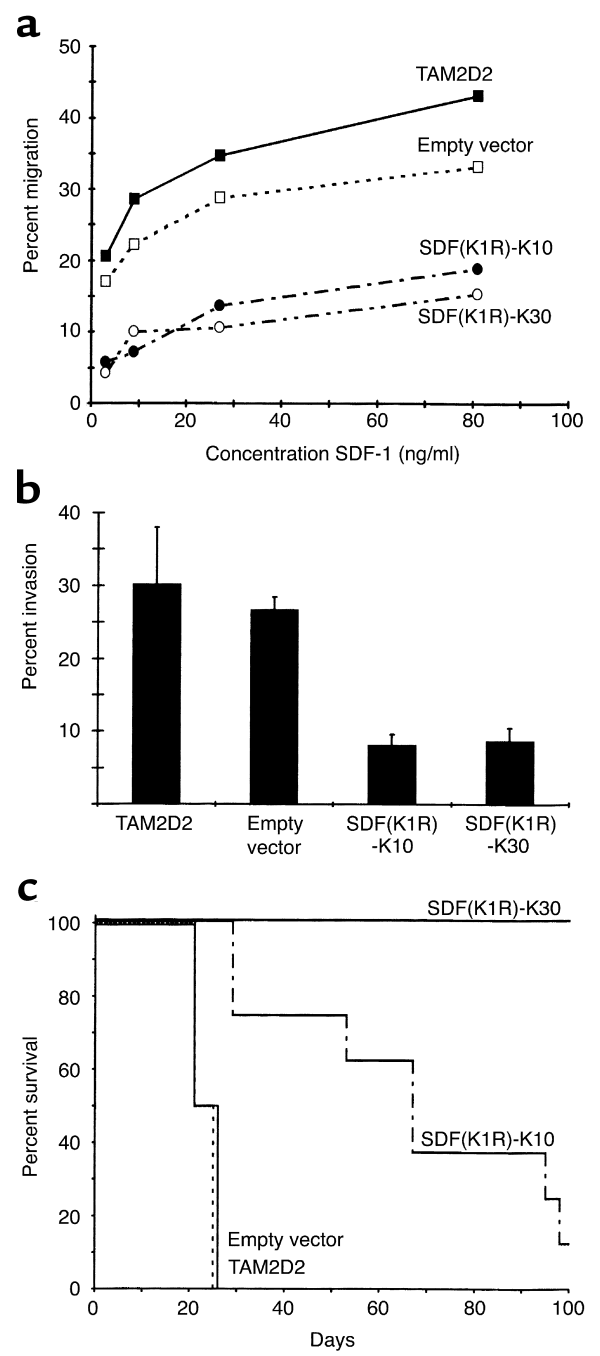

Figure 6

Migration, invasion, and dissemination of the SDF(K1R)-KDEL clones. Shown are the results of two of five clones, SDF(K1R)-K10 and SDF(K1R)-K30. (a) Data are the percentage of cells that have migrated in 2 hours through a filter to the lower chamber of a transwell containing different concentrations of SDF- 1 and are averages of five experiments performed. (b) Data are the percentages of cells that have invaded into the REF monolayer after 1 hour and are averages \pm SEM of six experiments performed. (c) Survival of syngeneic AKR mice injected intravenously with $5 \times 10^{5}$ cells is shown. Animals were killed when moribund or after 100 days. vival of all the mice injected with the SDF-1-KDEL transfectants suggests that SDF- 1 is involved in the dissemination to all of these tissues. Tashiro et al. (17) have shown that SDF-1 was constitutively expressed in all organs tested, but those did not include all dissemination sites. To determine the tissue distribution of SDF1 more extensively, we performed an RT-PCR on 14 different mouse tissues. As shown in Figure 8, SDF-1 was expressed in all organs. Therefore, SDF-1 is probably the only chemokine responsible for the dissemination of the $\mathrm{T}$ cell hybridoma cells to all the different tissues.

\section{Discussion}

We have used a $\mathrm{T}$ cell hybridoma to study mechanisms of in vivo migration into nonlymphoid tissues that are likely to be relevant for activated and memory $\mathrm{T}$ cells and possibly for the dissemination of certain types of lymphoma. In previous studies it was shown that this in vivo migration is blocked by pertussis toxin $(5,6)$, indicating the possible involvement of a chemokine. SDF-1 was a major candidate because, in contrast to many other chemokines that are expressed primarily at sites of inflammation and in hematopoietic tissues, SDF-1 is constitutively expressed in multiple nonhematopoietic organs. To demonstrate the involvement of SDF-1, we have reduced the surface level of its receptor by transfecting SDF-1 linked to the ER-retention signal KDEL $(23,24)$. Newly synthesized CXCR4 binds to this SDF-1$\mathrm{KDEL}$, is also retained in the $\mathrm{ER}$, and is thus prevented from reaching the cell surface. We generated a set of clones in which CXCR4 was completely eliminated from the cell surface, as shown by using a binding assay and confirmed by a complete loss of chemotactic response to SDF-1. This effect was specific since the cells still migrated in response to TARC. Furthermore, elimination of surface CXCR4 led to a complete loss of dissemination capacity. This implies that CXCR4 is involved in the in vivo migration of activated and memory $T$ cells into nonhematopoietic organs and potentially involved in the dissemination of lymphoma cells that express CXCR4.

At least 17 chemokine receptors are known to date, many of which bind multiple ligands. Consequently, a KDEL-conjugated chemokine may prevent more than one receptor from reaching the surface. However, so far SDF-1 is the only ligand known that binds to CXCR4 with high affinity $(34,35)$. Furthermore, the fact that SDF-1-deficient and CXCR4-deficient mice exhibit identical hematopoietic and cardiac failures $(36,37)$ also supports the notion that CXCR4 is the only SDF-1 receptor. Nevertheless, it cannot be completely excluded that a second SDF-1 receptor exists and is expressed by $T$ cells. This would only affect our conclusions if this hypothetical receptor would bind to other chemokines as well. The specificity of the effect of SDF-1-KDEL is demonstrated by the migration of the transfectants toward the chemokine TARC $(32,38)$, which was similar to that of the control cells. This shows that relevant signal pathways shared by the two chemokines were not affected by the introduction of the SDF-1-KDEL con- 


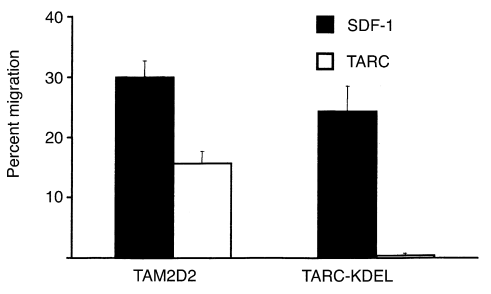

Figure 7

Migration of the TARC-KDEL transfectant. Data are the percentages of cells that have migrated in 2 hours through a filter to the lower chamber of a transwell containing $100 \mathrm{ng} / \mathrm{ml} \mathrm{SDF-1} \mathrm{or} \mathrm{TARC} \mathrm{and} \mathrm{are}$ averages of eight experiments performed.

struct or heterologous desensitization and that the migration machinery was still intact.

We also considered the possibility that binding of SDF-1-KDEL to CXCR4 induces signals that might cause the homologous downregulation of pathways not shared with TARC. Crump et al. showed that an SDF-1 mutant in which the N-terminal lysine of the mature peptide was changed to an arginine still binds CXCR4, but does not trigger signaling (33). We transfected an SDF-1-KDEL construct with this mutation into the $\mathrm{T}$ cell hybridoma. In this case, the expression levels obtained were not high enough to completely suppress CXCR4 function as shown by the reduction, but not loss, of SDF-1 binding. Nevertheless, both chemotaxis toward SDF-1 and invasion into fibroblast monolayers was substantially suppressed and also dissemination capacity was strongly reduced, arguing against an effect of SDF-1-KDEL-induced signals.

Another possibility is that introducing a protein with an ER retention signal (KDEL) inside the cell, has nonspecific effects due to the occupation of too many KDEL receptors. To exclude this possibility, another intrakine, TARC-KDEL, was transduced into the TAM2D 2 cells. This TARC-KDEL protein should retain CCR4, the receptor for TARC (32), inside the cell. Indeed, migration toward TARC was completely blocked, whereas migration toward SDF1 was not. Invasion into fibroblast monolayers of the TARC-KDEL transfectant was similar compared with the control cells. This was expected since invasion is completely dependent on CXCR4 signaling, and this was not blocked as shown in the migration assay. The TARC-KDEL transfectants were then examined for metastasis formation in vivo. Four out of 6 mice died within 6 weeks, at the same time and with the same pattern of metastases as the mice injected with control cells. The fact that these mice all had liver and spleen metastases and died at the same time as the control mice argues against a general KDEL effect. Thus, the block of dissemination as seen after injection of the SDF-K7 and SDF-K17 clones can be specifically assigned to the removal of CXCR4 from the surface and not to a general effect of the KDEL sequence.
The invasion into embryonic fibroblast monolayers was completely blocked in the SDF-1-KDEL transfectants, confirming the strong correlation of this invasion with dissemination of these cells as demonstrated repeatedly (4-7). These fibroblasts produce SDF-1 as tested by RT-PCR (data not shown), and apparently SDF-1 is present on the cell surface, probably associated with proteoglycans. To demonstrate that invasion is dependent on SDF-1 and that the lack of invasive capacity in the SDF-1-KDEL transfectants is only due to the loss of CXCR4, the fibroblasts were pretreated with TARC. We assumed that TARC would bind to the proteoglycans and induce invasion in the SDF-1-KDEL transfectants. Indeed, the cells invaded the TARC-pretreated monolayers, again showing that the required signal pathways were still intact. However, this invasion was relatively modest. Partly, this could be due to a weaker signal as shown by lower chemotaxis induced by the same high $(100 \mathrm{ng} / \mathrm{ml})$ concentrations of TARC and SDF- 1 . This could be caused by a lower surface level of the TARC receptor CCR4 $(38,39)$ compared with CXCR4. A more likely explanation is that the role of SDF- 1 in invasion is primarily the activation of the integrin LFA-1 (10). Although TARC can activate LFA-1 (40), it is clearly less effective than SDF-1 (41).

Dissemination was completely blocked in the SDF1-KDEL transfectants, showing that CXCR4 has a decisive role in the dissemination of $\mathrm{T}$ cell hybridomas. Further studies should reveal whether SDF-1 and other chemokines that are constitutively expressed, such as BCA-1 and LARC in the liver (19, 20 ), play a similar role in other hematopoietic malignancies. This notion is supported by our finding that dissemination of several hematopoietic tumors, but not all, is dependent on $G_{i}$ proteins (ref. 42; and I.S. Zeelenberg et al., unpublished results). However, each of these tumors expresses a different subset of chemokine receptors, so that multiple chemokines may be involved. Furthermore, it should be noted that the mere presence of a chemokine receptor is not sufficient, as described for germinal center B cells, which express CXCR4 but fail to migrate in response to

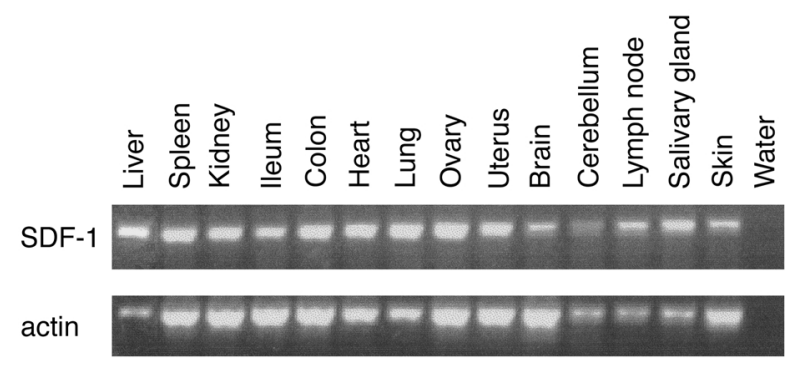

\section{Figure 8}

Expression of SDF-1 in different tissues of the mouse. SDF-1 is expressed in all tested tissues. Actin expression is shown as a control for the RT-PCR. 
SDF-1 until they differentiate (43). Similarly, we found that BW5147 cells, from which the T cell hybridomas were generated, express CXCR4 but fail to migrate toward SDF-1 (I.S. Zeelenberg et al., unpublished results).

The $\mathrm{T}$ cell hybridomas normally migrate to many tissues, and in all of these tissues this was blocked after the loss of CXCR4, implying that SDF-1 is involved in the migration to all these sites. Indeed, we show here that SDF- 1 is expressed in all tested tissues in addition to those described by Tashiro et al. (17). SDF-1 is also expressed in many tissues that are not (major) dissemination sites, such as heart, colon, and lymph nodes. Clearly, other factors such as adhesion molecules also influence dissemination patterns. A reason why the $\mathrm{T}$ cell hybridoma does not migrate to the colon could be that the integrin necessary for entry, $\alpha_{4} \beta_{7}$, is not present on these cells. Similarly, for migration into lymph nodes, cells have to migrate through high endothelium of postcapillary venules, and for this the cells need L-selectin (44). The lack of L-selectin on the surface of the T cell hybridomas (2) might thus explain the low tendency to migrate into lymph nodes.

CXCR 4 and CCR5 are the major coreceptors for HIV-1 entry $(22,26,27)$. The "intrakine" approach used in this study was in fact proposed as a tool for gene therapy of AIDS. Clinical trials are ongoing in which hematopoietic stem cells transduced with two intrakines, SDF-1-KDEL and RANTES-KDEL, are injected into patients (25). RANTES-KDEL binds to the chemokine receptor CCR5 and is expected to reduce surface levels similar to the way in which SDF-1-KDEL removes CXCR4 from the cell surface. Removal of CCR5 from the surface of lymphocytes conceivably would not be a problem since individuals with homozygous defects in CCR5 have no obvious deficiencies. This is probably due to the many ligands of CCR 5 that also bind to other receptors, so that the role of CCR5 can be taken over by other chemokine receptors (16). However, as stated above, SDF-1 is most likely the only ligand of CXCR4. We showed that removal of CXCR4 from the surface caused a complete block of in vivo migration of $\mathrm{T}$ cell hybridomas into many tissues, suggesting that lack of CXCR4 will disturb the normal in vivo migration patterns of activated and memory $\mathrm{T}$ cells. This might affect the effectiveness of the immune responses and may thus have consequences for the proposed gene therapy for AIDS, as well as other therapies aimed at blocking CXCR4.

\section{Acknowledgments}

We thank Ton Schrauwers and Henk Starreveld for excellent technical assistance in animal experiments and Martin van de Valk for microscopic examination of the animals. We are grateful to G.P. Nolan (Stanford University, Stanford, California, USA) for the pLZRS vector and $\Phi$ NXE cells, and G.A.M. Michiels, J.G. Col- lard, and R.D.M. Soede (Netherlands Cancer Institute, Amsterdam, The Netherlands) for modifications of the pLZRS vector. This work was supported by grant NKI 98-1679 from the Dutch Cancer Society, Amsterdam, The Netherlands.

1. Roos, E., La Riviere, G., Collard, J.G., Stukart, M.J., and De Baetselier, P. 1985. Invasiveness of T-cell hybridomas in vitro and their metastatic potential in vivo. Cancer Res. 45:6238-6243.

2. La Rivière, G., Gebbinck, J.W., Schipper, C.A., Mooi, W.J., and Roos, E. 1993. In vitro invasiveness of CTL clones and in vivo dissemination of CTL hybridomas. J. Leukoc. Biol. 53:381-389.

3. Mackay, C.R., Marston, W.L., and Dudler, L. 1990. Naive and memory T cells show distinct pathways of lymphocyte recirculation. J. Exp. Med. 171:801-817.

4. Roossien, F.F., de Rijk, D., Bikker, A., and Roos, E. 1989. Involvement of LFA-1 in lymphoma invasion and metastasis demonstrated with LFA-1deficient mutants. J.Cell Biol. 108:1979-1985.

5. Driessens, M.H.E., van Rijthoven, E.A.M., La Rivière, G., and Roos, E. 1996. Expression of pertussis toxin adenosine diphosphate-ribosyltransferase in a T-cell hybridoma reduces metastatic capacity. Blood. 88:3116-3123.

6. Soede, R.D.M., Wijnands, Y.M., Van Kouteren-Cobzaru, I., and Roos, E. 1998. ZAP-70 tyrosine kinase is required for LFA-1-dependent T cell migration. J.Cell Biol. 142:1371-1379.

7. Habets, G.G., et al. 1994. Identification of an invasion-inducing gene, Tiam-1, that encodes a protein with homology to GDP-GTP exchangers for Rho-like proteins. Cell. 77:537-549.

8. Springer, T.A. 1990. Adhesion receptors of the immune system. Nature. 346:425-434.

9. Lub, M., van Kooyk, Y., and Figdor, C.G. 1995. Ins and outs of LFA-1. Immunol. Today. 16:479-483.

10. La Rivière, G., Klein Gebbinck, J.W., Driessens, M.H.E., and Roos, E. 1994. Pertussis toxin inhibition of T-cell hybridoma invasion is reversed by manganese-induced activation of LFA-1. J.Cell Sci. 107:551-559.

11. Baggiolini, M., Dewald, B., and Moser, B. 1997. Human chemokines: an update. Annu. Rev. Immunol. 15:675-705.

12. Melchers, F., Rolink, A.G., and Schaniel, C. 1999. The role of chemokines in regulating cell migration during humoral immune responses. Cell. 99:351-354.

13. Schall, T.J., and Bacon, K.B. 1994. Chemokines, leukocyte trafficking, and inflammation. Curr. Opin. Immunol. 6:865-873.

14. Cyster, J.G. 1999. Chemokines and cell migration in secondary lymphoid organs. Science. 286:2098-2102.

15. Ward, S.G., and Westwick, J. 1998. Chemokines: understanding their role in T-lymphocyte biology. Biochem. J. 333:457-470.

16. Jung, S., and Littman, D.R. 1999. Chemokine receptors in lymphoid organ homeostasis. Curr. Opin. Immunol. 11:319-325.

17. Tashiro, K., et al. 1993. Signal sequence trap: a cloning strategy for secreted proteins and type I membrane proteins. Science. 261:600-603.

18. Nagasawa, T., Kikutani, H., and Kishimoto, T. 1994. Molecular cloning and structure of a pre-B-cell growth-stimulating factor. Proc. Natl. Acad. Sci. USA. 91:2305-2309.

19. Legler, D.F., et al. 1998. B cell-attracting chemokine 1, a human CXC chemokine expressed in lymphoid tissues, selectively attracts B lymphocytes via BLR1/CXCR5. J. Exp. Med. 187:655-660.

20. Baba, M., et al. 1997. Identification of CCR6, the specific receptor for a novel lymphocyte-directed CC chemokine LARC. J. Biol. Chem. 272:14893-14898.

21. Loetscher, M., et al. 1994. Cloning of a human seven-transmembrane domain receptor, LESTR, that is highly expressed in leukocytes. J. Biol. Chem. 269:232-237.

22. Feng, Y., Broder, C.C., Kennedy, P.E., and Berger, E.A. 1996. HIV-1 entry cofactor: functional cDNA cloning of a seven-transmembrane, $G$ protein-coupled receptor. Science. 272:872-877.

23. Chen, J.D., Bai, X., Yang, A.G., Cong, Y., and Chen, S.Y. 1997. Inactivation of HIV-1 chemokine co-receptor CXCR-4 by a novel intrakine strategy. Nat. Med. 3:1110-1116.

24. Bai, X., Chen, J.D., Yang, A.G., Torti, F., and Chen, S.Y. 1998. Genetic coinactivation of macrophage- and T-tropic HIV-1 chemokine coreceptors CCR-5 and CXCR-4 by intrakines. Gene Ther. 5:984-994.

25. Dorrell, S. 1999. Blocking HIV-1 infection with intrakines. Mol. Med. Today. 5:97.

26. Deng, H., et al. 1996. Identification of a major co-receptor for primary isolates of HIV-1. Nature. 381:661-666.

27. Dragic, T., et al. 1996. HIV-1 entry into CD4+ cells is mediated by the chemokine receptor CC-CKR-5. Nature. 381:667-673.

28. La Rivière, G., Schipper, C.A., Collard, J.G., and Roos, E. 1988. Invasiveness in hepatocyte and fibroblast monolayers and metastatic potential of T-cell hybridomas in mice. Cancer Res. 48:3405-3410.

29. Michiels, F., van der Kammen, R.A., Janssen, L., Nolan, G.P., and Collard, 
J.G. 2000. Expression of Rho GTPases using retroviral vectors. Methods Enzymol. 325:295-302.

30. Kinsella, T.M., and Nolan, G.P. 1996. Episomal vectors rapidly and stably produce high-titer recombinant retrovirus. Hum. Gene Ther. 7:1405-1413.

31. Mountford, P., et al. 1994. Dicistronic targeting constructs: reporters and modifiers of mammalian gene expression. Proc. Natl. Acad. Sci. USA. 91:4303-4307.

32. Imai, T., et al. 1996. Molecular cloning of a novel T cell-directed CC chemokine expressed in thymus by signal sequence trap using EpsteinBarr virus vector. J. Biol. Chem. 271:21514-21521.

33. Crump, M.P., et al. 1997. Solution structure and basis for functional activity of stromal cell-derived factor-1; dissociation of CXCR4 activation from binding and inhibition of HIV-1. EMBO J. 16:6996-7007.

34. Bleul, C.C., et al. 1996. The lymphocyte chemoattractant SDF-1 is a ligand for LESTR/fusin and blocks HIV-1 entry. Nature. 382:829-833.

35. Oberlin, E., et al. 1996. The CXC chemokine SDF-1 is the ligand for LESTR/fusin and prevents infection by T-cell-line-adapted HIV-1. Nature. 382:833-835.

36. Nagasawa, T., et al. 1996. Defects of B-cell lymphopoiesis and bone-marrow myelopoiesis in mice lacking the CXC chemokine PBSF/SDF-1. Nature. 382:635-638.

37. Zou, Y.R., Kottmann, A.H., Kuroda, M., Taniuchi, I., and Littman, D.R.
1998. Function of the chemokine receptor CXCR4 in haematopoiesis and in cerebellar. Nature. 393:595-599.

38. Imai, T., et al. 1997. The T cell-directed CC chemokine TARC is a highly specific biological ligand for CC chemokine receptor 4. J. Biol. Chem. 272:15036-15042.

39. Garlisi, C.G., et al. 1999. The assignment of chemokine-chemokine receptor pairs: TARC and MIP-1 beta are not ligands for human CCchemokine receptor 8. Eur. J. Immunol. 29:3210-3215.

40. Campbell, J.J., et al. 1999. The chemokine receptor CCR4 in vascular recognition by cutaneous but not intestinal memory T cells. Nature. 400:776-780.

41. Campbell, J.J., et al. 1998. Chemokines and the arrest of lymphocytes rolling under flow conditions. Science. 279:381-384.

42. Soede, R.D., Wijnands, Y.M., Kamp, M., Der Valk, M.A., and Roos, E. 2000. $\mathrm{Gi}$ and $\mathrm{Gq} / 11$ proteins are involved in dissemination of myeloid leukemia cells to the liver and spleen, whereas bone marrow colonization involves $\mathrm{Gq} / 11$ but not Gi. Blood. 96:691-698.

43. Bleul, C.C., Schultze, J.L., and Springer, T.A. 1998. B lymphocyte chemotaxis regulated in association with microanatomic localization, differentiation state, and B cell receptor engagement. J. Exp. Med. 187:753-762.

44. Kim, C.H., and Broxmeyer, H.E. 1999. Chemokines: signal lamps for trafficking of T and B cells for development and effector function. J. Leukoc. Biol. 65:6-15. 